\title{
Antioxidant and Hepatoprotective Effect of Swertiamarin on Carbon Tetrachloride- Induced Hepatotoxicity via the Nrf2/HO-1 Pathway
}

\author{
Tao Wu Jingjing Li Yong Li Hongping Song \\ Department of Pharmacy, Puai Hospital, Tongji Medical College, Huazhong University of Science and \\ Technology, Wuhan, China
}

\section{Key Words}

Swertiamarin $\bullet$ Carbon tetrachloride $\bullet \mathrm{Nrf2} \bullet$ Oxidative stress $\bullet$ Inflammation $\bullet$ Efflux transporters

\begin{abstract}
Background/Aims: Swertiamarin (STM), the main bioactive component in Swertia mussotii Franch (Gentianaceae), has been shown to exert hepatoprotective effects on experimental liver injury. However, the effects and exact mechanisms of STM on carbon tetrachloride $\left(\mathrm{CCl}_{4}\right)$ causing hepatotoxicity are still unknown. This study investigated the potential protective effects and mechanisms of STM on $\mathrm{CCl}_{4}$-induced liver injury in rats. Methods: Adult male Sprague-Dawley (SD) rats were exposed to $\mathrm{CCl}_{4}$ with or without STM co-administration for consecutive eight weeks. Results: STM significantly ameliorated $\mathrm{CCl}_{4}$-induced increase in serum alanine aminotransferase (ALT), aspartate aminotransferase (AST) and alkaline phosphatase (ALP) levels and histopathological changes in the liver. Hepatic oxidative stress was repressed by STM, as evidenced by the decrease in malondialdehyde (MDA), with concomitant increase in antioxidase activity (e.g. superoxide dismutase (SOD); glutathione peroxidase (GPx)), glutathione (GSH) level. STM also obviously attenuated inflammatory response in $\mathrm{CCl}_{4}$-lesioned livers as evidenced by the decrease in inflammatory cytokines/ chemokines (e.g. inducible nitric oxide synthase (iNOS), interleukin-1 $\beta$ (IL-1ß)). Additionally, STM significantly induced the expression of CYPs, efflux transporters and PDZK1 as compared with the $\mathrm{CCl}_{4}$ group. Moreover, co-administration of STM with $\mathrm{CCl}_{4}$ remarkably up-regulated the expression of Nrf2, $\mathrm{HO}-1$ and NQO1 compared with the $\mathrm{CCl}_{4}$ group. Conclusions: The present study demonstrates that STM exerts a protective effect against $\mathrm{CCl}_{4}$-induced liver injury and inflammation with its antioxidant effects and induction of hepatic detoxification enzymes and efflux transporters expression, at least in part, via the $\mathrm{Nrf2} / \mathrm{HO}-1$ pathway in rats.
\end{abstract}

\section{Introduction}

2017 The Author(s)

Published by S. Karger AG, Basel

Swertiamarin (STM) is the main bioactive component in Swertia mussotii Franch (Gentianaceae), a commonly used Tibetan medicine, exerting broad pharmacological 
activities, especially hepatoprotective effect [1-3]. It has been previously reported to attenuate experimental hepatic fibrosis by suppressing the rennin-angiotensin system [4]. However, the effects and mechanisms of STM on carbon tetrachloride $\left(\mathrm{CCl}_{4}\right)$-induced liver injury remain unclear.

Carbon tetrachloride $\left(\mathrm{CCl}_{4}\right)$ is a well-known hepatotoxin that is widely used to induce experimental liver fibrosis and cirrhosis in rodents [5]. $\mathrm{CCl}_{4}$-induced hepatotoxicity is believed to involve two phases. The initial phase involves the metabolism of $\mathrm{CCl}_{4}$ by cytochrome $\mathrm{P} 450$ (CYP) to the trichloromethyl radicals $\left(\bullet \mathrm{CCl}_{3}\right.$ and/or $\left.\mathrm{CCl}_{3} \mathrm{OO} \bullet\right)$, which lead to membrane lipid peroxidation and finally to cell necrosis [6,7]. The second phase of $\mathrm{CCl}_{4}{ }^{-}$ induced hepatotoxicity involves the activation of Kupffer cells, which is accompanied by the production of proinflammatory factors [8]. Thus, $\mathrm{CCl}_{4}$ at a high dosage can cause liver injury and fibrosis through cellular necrosis, oxidative stress and inflammation.

It is well known that cell damage induced by reactive oxygen species (ROS) is an important mechanism of liver injury. Naturally, cells are induced to develop some adaptive responses by intrinsic and extrinsic oxidants and electrophiles to counteract the environmental stresses [9]. The redox sensitive transcription factor, nuclear E2-related factor 2 (Nrf2) is an essential transcription factor that regulates an array of detoxifying and antioxidant defense genes expression through the antioxidant response element (ARE) in the liver [10]. Nrf2 controls the basal and induced expression of hundreds of ARE-dependent genes to regulate the physiological and pathophysiological outcomes of oxidant exposure. Many researchers have demonstrated that Nrf2 prevented the liver from kinds of hepatotoxicants and Nrf2 signaling pathway represented an important target in protecting the liver against the damage induced by ethanol [11-13].

In this study, we mainly focused on the protective effects of STM against $\mathrm{CCl}_{4}$-induced liver injury. Furthermore, we measured the expression of $\mathrm{Nrf} 2$ and some detoxifying enzymes (HO-1, NQ01) to elucidate the antioxidant pathway of STM.

\section{Materials and Methods}

\section{Reagents and antibodies}

Swertiamarin (purity $>98 \%$, dissolved in 1\% Tween-20 saline) was provided by Xi'an Jiatian Biotechnology Co., Ltd (Xi'an, China). Carbon tetrachloride was purchased from Sinopharm Chemical Reagent Co., Ltd (Shanghai, China). Anti-CYP2E1 antibody, anti-CYP3A antibody, anti-Bsep antibody, antiMrp2 antibody, anti-Mrp3 antibody, anti-Mrp4 antibody, anti-PDZK1 antibody, anti-Nrf2 antibody, antiNQO1 antibody and anti-HO-1 antibody were purchased from Santa Cruz Biotechnology Inc (California, USA). Anti-GAPDH antibody was purchased from Beijing Biosynthesis Biotechnology., Ltd (Beijing, China). Anti-Histone antibody was purchased from Santa Cruz Biotechnology Inc (California, USA). BCA assay kit was purchased from Beyotime Institute of Biotechnology (Shanghai, China).

\section{Animals and experimental protocol}

This study was carried out in strict accordance with the guideline of the Council on Animal Care of Academia Sinica. The protocol was approved by the Ethical Committee on Animal Experimentation of Puai Hospital, Huazhong University of Science and Technology, China. Adult male SD rats weighing 250-280 g were obtained from the Center of Experimental Animal of Hubei Province (Wuhan, China). All animals were kept under the same laboratory conditions of temperature $\left(25 \pm 2^{\circ} \mathrm{C}\right)$ and lighting $(12: 12 \mathrm{~h}$ light: dark cycle), and were given free access to standard laboratory chow and tap water. All rats were allowed to acclimatize for one week before experiment. The animals were randomly assigned to five experimental groups $(n=12)$, namely:

i. Control group, rats were given $1 \%$ Tween-20 saline by gavage once per day for consecutive eight weeks with co-administration of vehicle (peanut oil, solvent of Carbon tetrachloride, $0.3 \mathrm{~mL} / 100 \mathrm{~g}$, s. c. twice a week).

ii. STM $200 \mathrm{mg} / \mathrm{kg}$ group, rats were given STM dissolved in $1 \%$ Tween-20 saline (200 mg/kg B.W.) by gavage once per day for consecutive eight weeks with co-administration of vehicle (peanut oil, solvent of 


\section{Cellular Physiology Cell Physiol Biochem 2017;41:2242-2254 and Biochemistry Publisher onIIne: Aprl 24, $2017 \quad \begin{aligned} & \text { DOI: 10.1159/000475639 } 2017 \text { The Author(s). Published by S. Karger AG, Basel } \\ & \text { www.karger.com/cpb }\end{aligned}$}

Wu et al.: Swertiamarin Protects Against Hepatotoxicity

Carbon tetrachloride, $0.3 \mathrm{~mL} / 100 \mathrm{~g}$, s. c. twice a week).

iii. $\mathrm{CCl}_{4}$ group, rats were given $1 \%$ Tween-20 saline by gavage once per day for consecutive eight weeks with co-administration of $40 \% \mathrm{CCl}_{4}$ mixed peanut oil solution $(0.3 \mathrm{~mL} / 100 \mathrm{~g}$, s. c. twice a week).

iv. $\mathrm{CCl}_{4}+\mathrm{STM} 100 \mathrm{mg} / \mathrm{kg}$ group, rats were treated with STM dissolved in $1 \%$ Tween-20 saline $(100$ $\mathrm{mg} / \mathrm{kg}$ B.W.) by gavage once per day for consecutive eight weeks with co-administration of $40 \% \mathrm{CCl}_{4}$ mixed peanut oil solution $(0.3 \mathrm{~mL} / 100 \mathrm{~g}$, s. c. twice a week).

v. $\mathrm{CCl}_{4}+\mathrm{STM} 200 \mathrm{mg} / \mathrm{kg}$ group, rats were treated with STM dissolved in $1 \%$ Tween-20 saline $(200 \mathrm{mg} /$ kg B.W.) by gavage once per day for consecutive eight weeks with co-administration of $40 \% \mathrm{CCl}_{4}$ mixed peanut oil solution $(0.3 \mathrm{~mL} / 100 \mathrm{~g}$, s. c. twice a week).

Rats were fasted for $12 \mathrm{~h}$ after the last dose of agents before they were anaesthetized with pentobarbital sodium (30 mg/kg B.W., intraperitoneally). The blood samples were collected from abdominal aorta for the assays of serum enzymes. One piece of the liver was immediately fixed in $10 \%$ formaldehyde for histopathology assay and immunostaining. The rest was frozen with liquid nitrogen, and stored at $-80^{\circ} \mathrm{C}$ until use.

\section{Histological evaluations}

Liver tissues were fixed with $10 \%$ formalin for $24 \mathrm{~h}$, dehydrated, and embedded in paraffin. The paraffin-embedded samples were sectioned at $5 \mu \mathrm{m}$ thickness and processed for hematoxylin-eosin (H\&E) and Masson's trichrome staining.

\section{Assessment of hepatotoxicity}

The blood samples were centrifuged at $4{ }^{\circ} \mathrm{C}$ for 15 minutes to separate serum. Liver enzymes alanine aminotransferase (ALT), aspartate aminotransferase (AST) and alkaline phosphatase (ALP) were estimated in serum by using commercial test kit (Jiancheng Institute of Biotechnology, Nanjing, China) according to the manufacturer's instructions.

\section{Liver oxidative damage assay}

Malondialdehyde (MDA), a degrading product of lipid peroxidation, was determined according to the thiobarbituric acid method using a MDA test kit (Jiancheng Institute of Biotechnology, Nanjing, China). Liver glutathione (GSH), a non-enzymatic antioxidant of hepatic antioxidant defense system, was assayed using commercial test kit (Jiancheng Institute of Biotechnology, Nanjing, China) according to the manufacturer's instructions.

\section{Liver inflammation assessment}

The level of myeloperoxidase (MPO), inducible nitric oxide synthase (iNOS) and nitric oxide (NO) were determined by using commercially available kits (Jiancheng Institute of Biotechnology, Nanjing, China). The level of interleukin-1 $\beta$ (IL-1 $\beta$ ), interleukin-6 (IL-6) and tumor necrosis factor alpha (TNF- $\alpha$ ) were determined by using ELISA kits (Neobioscience, Beijing, China) according to the manufacturer's instructions.

\section{Hepatic antioxidative defense potentials}

Hepatic antioxidative defense enzymes including superoxide dismutase (SOD), catalase (CAT), glutathione peroxidase (GPx) and glutathione-S-transferase (GST) were assayed using commercial test kits (Nanjing Jiancheng Bioengineering Institute, China).

\section{Western blotting}

The protein levels of detoxification enzymes, efflux transporters and Nrf2 were determined in the liver by western blotting analysis. The whole liver lysate was prepared to evaluate the expression level of CYP2E1, CYP3A, PDZK1, HO-1 and NQ01. The membrane protein fractions of liver tissue were prepared to investigate the levels of Bsep, Mrp2, Mrp3 and Mrp4. The nuclear extracts of liver tissue were prepared to evaluate the expression level of Nrf2 in nucleus. The protein concentration was determined using the bicinchoninic acid (BCA) assay. Equal amounts of protein were separated with $10 \%$ sodium dodecyl sulfate (SDS)-polyacrylamide gel electrophoresis. After electrophoresis, the gels were transferred onto polyvinylidene difluoride membranes, which were blocked with Tris-buffered saline containing $5 \%$ nonfat milk at $4^{\circ} \mathrm{C}$. Then, the membranes were incubated overnight at $4^{\circ} \mathrm{C}$ in solution containing $0.1 \%$ Tween 20 , 


\section{Cellular Physiology Cell Physiol Biochem 2017;41:2242-2254 and Biochemistry Publisned onIIne: AprII 24, $2017 \quad \begin{aligned} & \text { DOI: 10.1159/000475639 } \\ & \begin{array}{l}\text { c } 2017 \text { The Author(s). Published by S. Karger AG, Basel } \\ \text { ww.karger.com/cpb }\end{array}\end{aligned}$ \\ Wu et al.: Swertiamarin Protects Against Hepatotoxicity}

5\% nonfat milk and the following primary antibodies: CYP2E1 (1:500); CYP3A (1:500); PDZK1 (1:500); HO-1 (1:500); NQO1 (1:500); Bsep (1:500); Mrp2 (1:500); Mrp3 (1:500); Mrp4 (1:500); Nrf2 (1:500); GAPDH (1:20000); Histon H3 (1:1000). Immunoreactive bands were quantified using the Gel-Pro Analyzer software (Media Cybernetics, Bethesda, MD). GAPDH or Histone H3 was used as an internal index.

\section{Statistical Analysis}

Data were expressed as mean \pm S.D. The significant differences between groups were assessed with SPSS version 13.0. The differences between group means were calculated by one-way ANOVA with LSD post hoc analysis. Difference was considered statistically significant when $p<0.05$, and extremely significant when $p<0.01$.

\section{Results}

\section{Effect of STM on histopathology of liver}

The hepatic samples of the control and STM 200 groups presented a normal cellular architecture with hepatocytes in ordered arrangement. However, liver tissues from rats treated with $\mathrm{CCl}_{4}$ showed extensive histopathological changes, characterized by hepatic lobule impairment, severe hepatocytes degeneration/necrosis, fatty changes, inflammatory cell infiltration and congestion. The histopathological lesions in the $\mathrm{CCl}_{4}+\mathrm{STM}$ groups were improved notably compared with those in the $\mathrm{CCl}_{4}$ group. This indicated that STM attenuated the intensity of $\mathrm{CCl}_{4}$-induced liver damage (Fig. 1A).

To confirm hepatic fibrosis histologically, a Masson's trichrome staining was performed (Fig. 1B). The staining revealed that $\mathrm{CCl}_{4}$ increased hepatic fibrosis when compared with the control group. And hepatic fibrosis was attenuated by STM administration at 100 and $200 \mathrm{mg} / \mathrm{kg}$.

\section{Effect of STM on liver injury markers}

To evaluate the effects of STM on liver injure induced by $\mathrm{CCl}_{4}$ in rats, the serum levels of ALT, AST and ALP were measured. As shown in Figure 2A-C, the levels of ALT, AST and ALP were significantly $(p<0.01)$ increased in the $\mathrm{CCl}_{4}$-treated group. After the administration of STM, there were a significant reduction in ALT, AST and ALP $(p<0.05$ or $p<0.01)$ compared
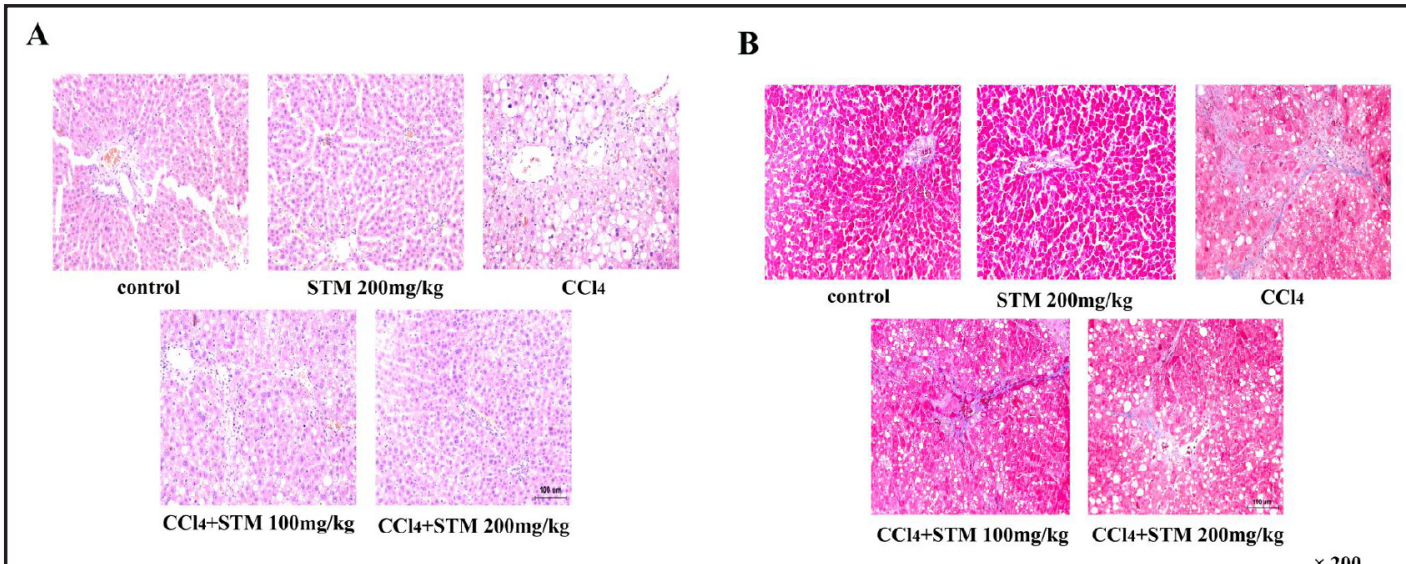

Fig. 1. Histological images of liver samples. Control and STM 200mg/kg: normal liver histology without fibroplasia and inflammatory cell infiltration; $\mathrm{CCl}_{4}$ : The normal structure of hepatic lobules was damaged with many inflammatory cells infiltration; hepatocytes degeneration/necrosis, fatty changes, infiltration of lymphocytes and severe collagen fibers presented with pseudo-lobe were observed; $\mathrm{CCl}_{4}+\mathrm{STM} 100 \mathrm{mg} / \mathrm{kg}$ : comparatively low degree of fibrotic changes with STM treatment (100 mg/kg B.W.); $\mathrm{CCl}_{4}+\mathrm{STM} 200 \mathrm{mg} / \mathrm{kg}$ : relieved liver histopathology with STM treatment (200 mg/kg B.W.). (A. H-E; B. Masson, original magnification $\times 200$ ). 


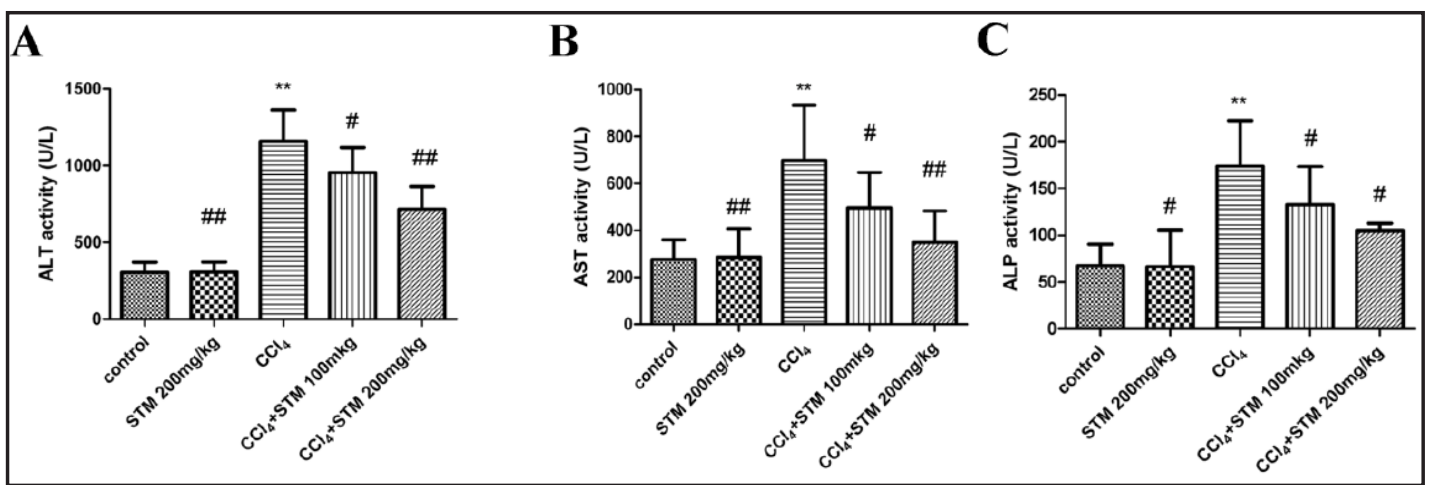

Fig. 2. Effect of STM on $\mathrm{CCl}_{4}$-induced elevations in serum liver enzyme activities in rats. A. ALT in $\mathrm{CCl}_{4}$-treated rats with different doses of STM for four weeks. B. AST in $\mathrm{CCl}_{4}$-treated rats with different doses of STM for eight weeks. C. ALP in $\mathrm{CCl}_{4}$-treated rats with different doses of STM for eight weeks. Data are represented as means \pm S.D. for 7-12 animals per group. ${ }^{*} p<0.05$ versus control, ${ }^{* *} p<0.01$ versus control; ${ }^{\#} p<0.05$ versus $\mathrm{CCl}_{4}$, , $p<0.01$ versus $\mathrm{CCl}_{4}$ by one- way ANOVA and LSD post hoc test.

Fig. 3. Effects of STM on $\mathrm{CCl}_{4}$-induced hepatic oxidative stress. A. Level of MDA. B. Level of GSH. C. Level of SOD. D. Level of CAT. E. Level of GPx. F. Level of GST. Data are represented as means \pm S.D. for 7-12 animals per group. ${ }^{*} p<0.05$ versus control, ${ }^{* *} p<0.01$ versus control; \# $p<0.05$ versus $\mathrm{CCl}_{4}$, \#\# $p<0.01$ versus $\mathrm{CCl}_{4}$ by one- way ANOVA and LSD post hoc test.

\section{A}

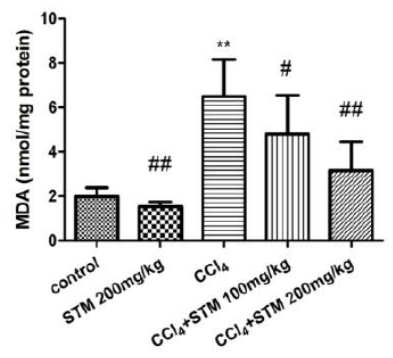

C

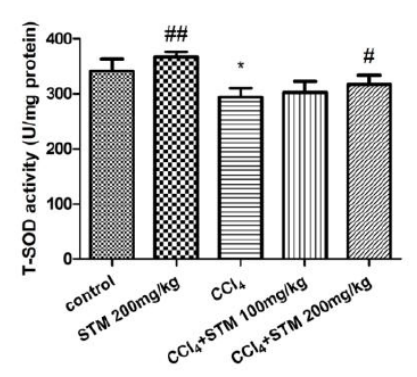

$\mathbf{E}$

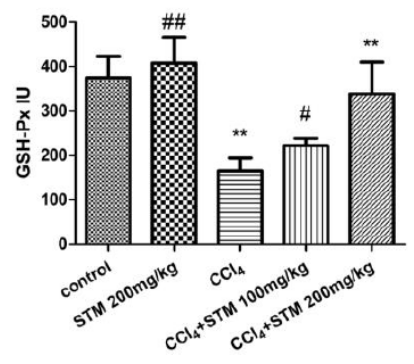

B

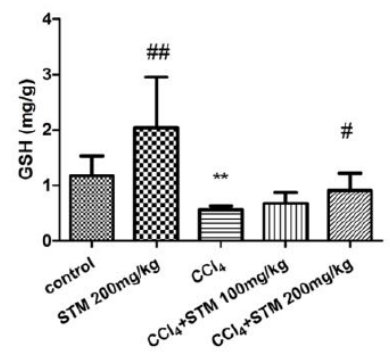

D

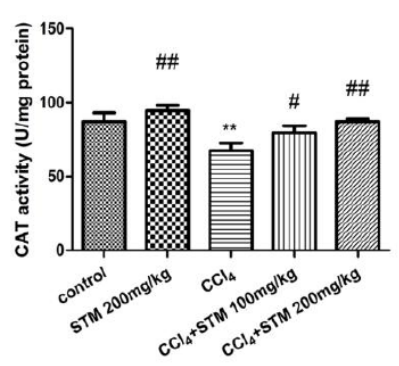

$\mathbf{F}$

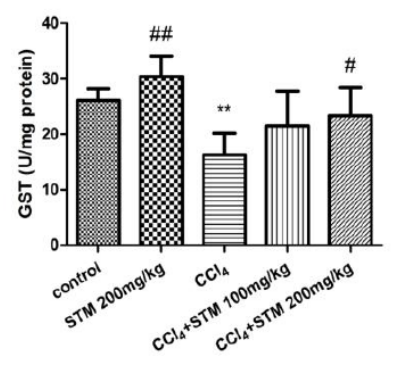

with the $\mathrm{CCl}_{4}$ group. Liver injure seemed to attenuate more frequently in rats treated with higher concentrations of STM than that with a lower one. 
Fig. 4. Effect of STM on inflammatory response in liver A. Level of MPO. B. Level of iNOS. C. Level of NO. D. Level of IL-1ß. E. Level of IL-6. F. Level of TNF- $\alpha$. Data are represented as means \pm S.D. for 7-12 animals per group. ${ }^{*} p<0.05$ versus control, ${ }^{* *} p<0.01$ versus control; \# $p<0.05$ versus $\mathrm{CCl}_{4}$, \#\# $p<0.01$ versus $\mathrm{CCl}_{4}$ by one- way ANOVA and LSD post hoc test.

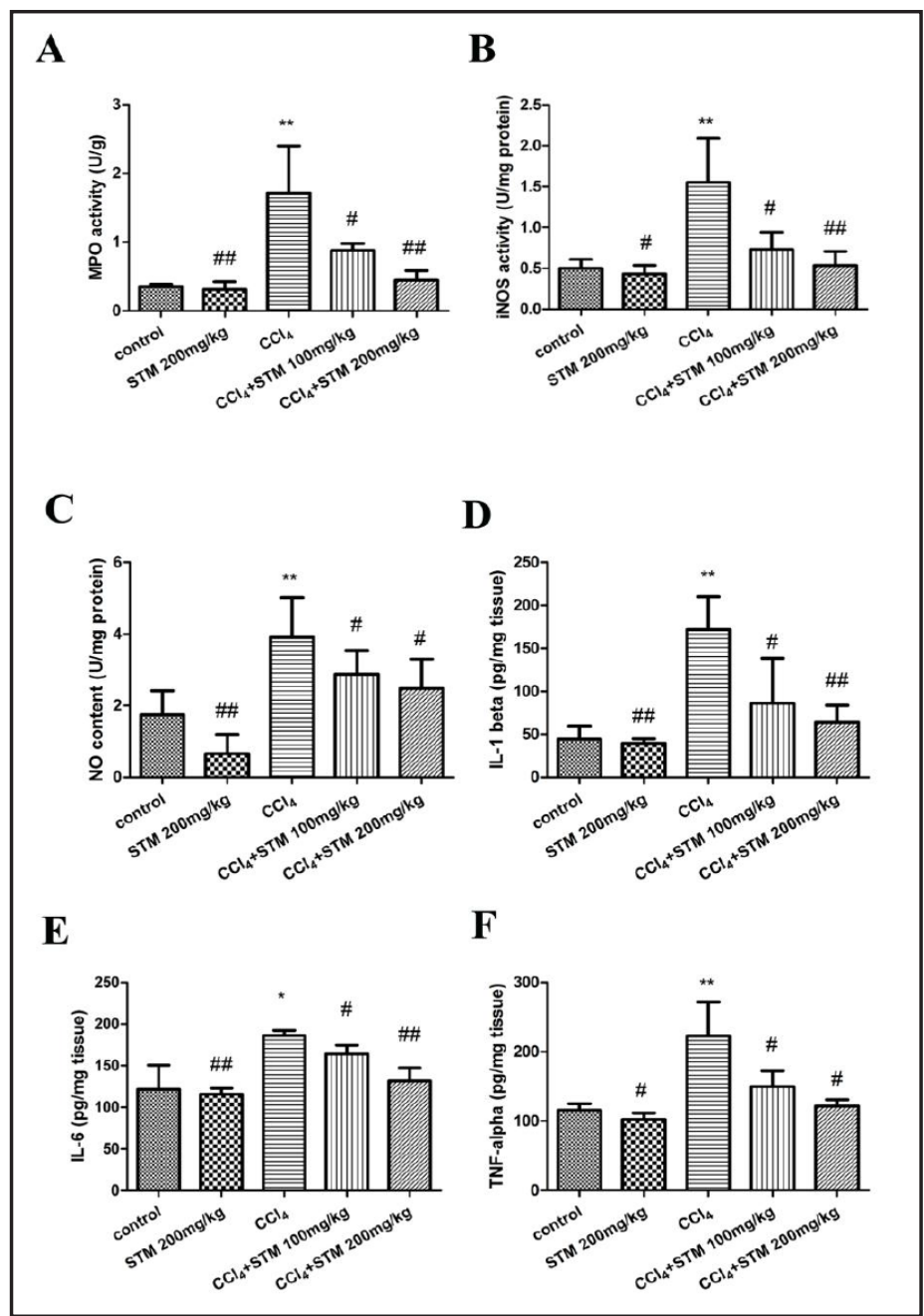

Effect of STM on oxidative stress in liver

MDA is the marker of lipid peroxidation and end product of oxidative decomposition of polyunsaturated fatty acid. The effects on the levels of MDA in the liver of different groups are shown in Figure 3A. CCl significantly $(p<0.01)$ increased the hepatic MDA levels in the $\mathrm{CCl}_{4}$ group. The STM treatment at higher as well as lower doses significantly lowered the MDA levels in the liver tissue in comparison with the $\mathrm{CCl}_{4}$ group.

$\mathrm{CCl}_{4}$ treatment significantly decreased the levels of GSH and SOD in the liver tissue as compared to the control group ( $p<0.01$ and $p<0.05$ ). STM treatment significantly ( $p<$ 0.05 ) increased the GSH levels at $200 \mathrm{mg} / \mathrm{kg}$ dose in comparison with the $\mathrm{CCl}_{4}$ group. The SOD levels were also improved significantly $(p<0.05)$ by STM treatment at $200 \mathrm{mg} / \mathrm{kg}$ in comparison with the $\mathrm{CCl}_{4}$ group (Fig. 3B, C).

$\mathrm{CCl}_{4}$ treatment significantly $(p<0.01)$ decreased the activities of CAT, GPx and GST in the liver tissue as compared to the control group. STM treatment markedly restored the activities of CAT, GPx at 100 as well as $200 \mathrm{mg} / \mathrm{kg}$ dose in comparison with the $\mathrm{CCl}_{4}$ group ( $p$ $<0.05$ or $p<0.01)$. And STM significantly $(p<0.05)$ increased the activity of GST at $200 \mathrm{mg} /$ $\mathrm{kg}$ in comparison with the $\mathrm{CCl}_{4}$ group (Fig. 3D, E, F).

\section{Effect of STM on inflammatory response in liver}

The results showed that MPO activity in the $\mathrm{CCl}_{4}$-treated group, an index of neutrophil infiltration, was increased significantly compared with that of the control group (Fig. 4A). Following STM $(100$ and $200 \mathrm{mg} / \mathrm{kg}$ ) treatment, MPO activity was decreased remarkably as compared to the $\mathrm{CCl}_{4}$ group $(p<0.01)$. The activity of iNOS was increased in the $\mathrm{CCl}_{4}$ group 


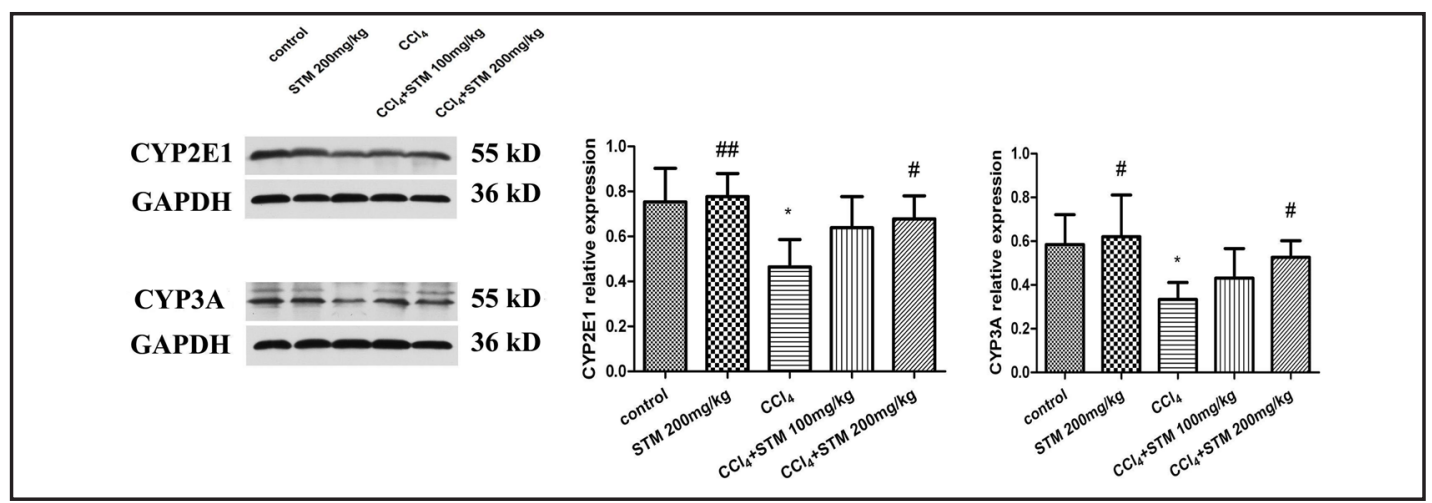

Fig. 5. Effect of STM on detoxification enzymes expression in liver. A. CYP2E1 in $\mathrm{CCl}_{4}$-treated rats with different doses of STM for eight weeks. B. CYP3A in $\mathrm{CCl}_{4}$-treated rats with different doses of STM for eight weeks. Data are represented as means \pm S.D. for 3-4 animals per group. ${ }^{*} p<0.05$ versus control, ${ }^{* *} p<0.01$ versus control; ${ }^{*} p<0.05$ versus $\mathrm{CCl}_{4}$, , $p<0.01$ versus $\mathrm{CCl}_{4}$ by one- way ANOVA and LSD post hoc test.

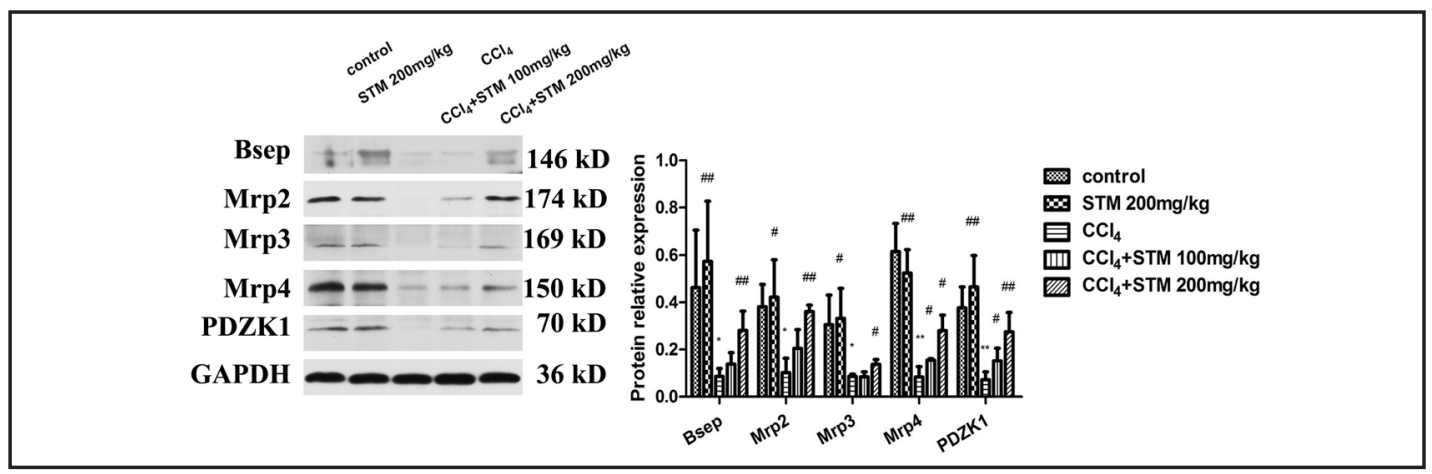

Fig. 6. Effect of STM on efflux transporters expression in liver. A. Level of Bsep. B. Level of Mrp2. C. Level of Mrp3. D. Level of Mrp4. E. Level of PDZK1. Data are represented as means \pm S.D. for 3-4 animals per group. ${ }^{*} p<0.05$ versus control, ${ }^{* *} p<0.01$ versus control; ${ }^{\#} p<0.05$ versus $\mathrm{CCl}_{4}$, ${ }^{\# \#} p<0.01$ versus $\mathrm{CCl}_{4}$ by one- way ANOVA and LSD post hoc test.

obviously compared to the control group ( $p<0.01$ ) (Fig. 4B). In the treatment group with $\mathrm{STM}$ and $\mathrm{CCl}_{4}$, the activity of iNOS was decreased markedly compared to the $\mathrm{CCl}_{4}$ group $(p$ $<0.01)$. In addition, $\mathrm{CCl}_{4}$ also elevated the hepatic NO level notably $(p<0.01)$ compared with that of the control group (Fig. 4C). The hepatic NO level was significantly decreased following treatment with STM compared to the $\mathrm{CCl}_{4}$ group $(p<0.01)$.

The content of pro-inflammatory cytokines, IL-1 $\beta$, IL- 6 and TNF- $\alpha$ in the liver displays the degree of inflammatory response in liver. The IL-1 $\beta$, IL- 6 and TNF- $\alpha$ content in liver tissue in STM-treated groups was significantly lower than that in the $\mathrm{CCl}_{4}$ group $(p<0.01)$, indicating the alleviation of liver inflammation with STM treatment (Fig. 4D-F).

\section{Effect of STM on detoxification enzymes expression in liver}

Western blot analysis was performed to examine the effect of STM on CYP2E1 and CYP3A protein expression. Compared to the control rats, rats receiving $\mathrm{CCl}_{4}$ alone showed a remarkable decrease in CYP2E1 protein level $(p<0.05)$. In rats co-treated with $200 \mathrm{mg} /$ $\mathrm{kg} \mathrm{STM}$, an obvious higher level of CYP2E1 $(p<0.05)$ was found as compared with the rats intoxicated with $\mathrm{CCl}_{4}$ alone. The restoration of CYP2E1 protein content by STM treatment implied that STM could have a hepatoprotective effect, which led to rapid recovery from $\mathrm{CCl}_{4}$-induced liver injury. Western blotting results also demonstrated that the protein level of CYP3A was significantly reduced by $\mathrm{CCl}_{4}$ intoxication $(p<0.05)$. STM $(200 \mathrm{mg} / \mathrm{kg})$ treatment elevated the protein level of CYP3A notably $(p<0.05)$ compared with the $\mathrm{CCl}_{4}$ only group (Fig. 5). 


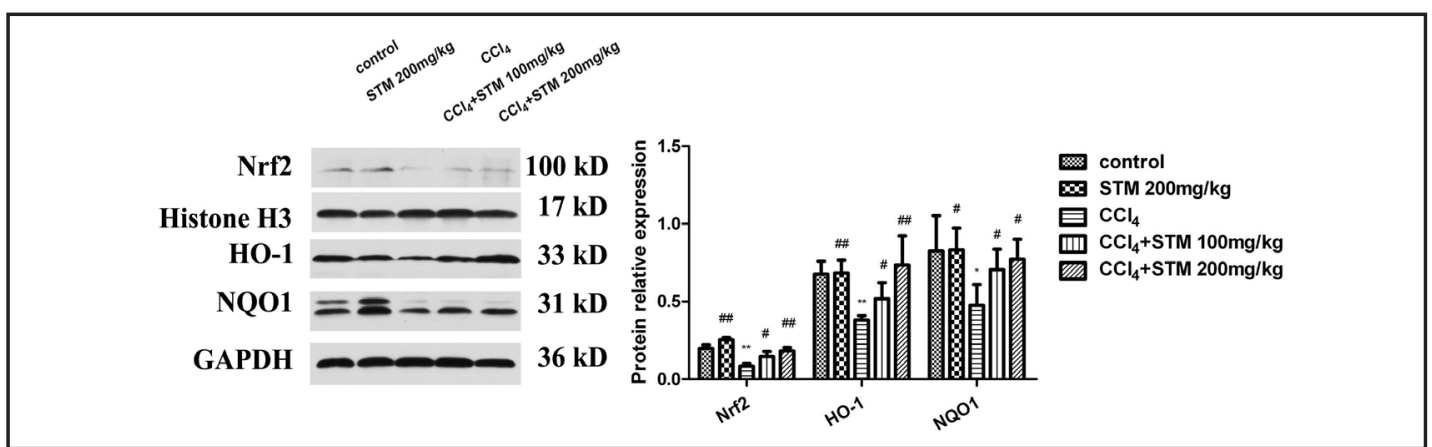

Fig. 7. Effects of STM on $\mathrm{CCl}_{4}$-induced hepatic Nrf2/HO-1 pathway. A. Relative density analysis of the Nrf2 protein bands in nucleus. B. Relative density analysis of HO-1. C. Relative density analysis of NQ01. GAPDH and Histon $\mathrm{H} 3$ were probed as the internal control in relative density analysis. Data are represented as means \pm S.D. for 3-4 animals per group. ${ }^{*} p<0.05$ versus control, ${ }^{* *} p<0.01$ versus control; ${ }^{\#} p<0.05$ versus $\mathrm{CCl}_{4^{\prime}}$ $\#$ \#\# $p<01$ versus $\mathrm{CCl}_{4}$ by one- way ANOVA and LSD post hoc test.

\section{Effect of STM on efflux transporters expression in liver}

$\mathrm{CCl}_{4}$ intoxication reduced the expression of canalicular Bsep and Mrp2 significantly compared with the control group $(p<0.05)$. The protein levels of Bsep and Mrp2 at the canalicular membrane of hepatocytes were markedly increased in $\mathrm{CCl}_{4}+\mathrm{STM} 200 \mathrm{mg} / \mathrm{kg}$ group compared with the $\mathrm{CCl}_{4}$ group $(p<0.01)$. $\mathrm{CCl}_{4}$ significantly reduced the expression of basolateral Mrp3 and Mrp4 $(p<0.05$ and $p<0.01)$. The protein levels of Mrp3 and Mrp4 were notably elevated in $\mathrm{CCl}_{4}+\mathrm{STM}$ groups compared with the $\mathrm{CCl}_{4}$ group. On the other hand, $\mathrm{CCl}_{4}$ repressed the protein expression of PDZK1 markedly $(p<0.01)$ compared with the control group. And the protein expression of PDZK1 was remarkably up-regulated in $\mathrm{CCl}_{4}+\mathrm{STM}$ groups compared with the $\mathrm{CCl}_{4}$ group (Fig. 6).

\section{Effect of STM on Nrf2/HO-1 pathway in liver}

The accumulated evidence showed that Nrf2 is a basic leucine zipper redox-sensitive transcriptional factor that plays a center role in ARE-mediated induction of phase II detoxifying and antioxidant enzymes. To further investigate the molecular mechanism of oxidative stress in rat liver, we measured the expression levels of $\mathrm{Nrf} 2$, heme oxygenase-1 (HO-1) and NAD(P)H: quinone oxidoreductase-1 (NQ01). Nrf2 expression levels in nuclear fractions were significantly decreased in $\mathrm{CCl}_{4}$-treated rats as compared with the controls $(p<0.01)$. Co-treatment with STM $(100$ an $200 \mathrm{mg} / \mathrm{kg})$ strongly increased the translocation of Nrf2 from the cytosol to the nuclear fraction compared with the $\mathrm{CCl}_{4}$ group. The levels of $\mathrm{HO}-1$ and NQO1 were decreased in rat treated with $\mathrm{CCl}_{4}$ alone as compared with controls $(p<0.01$ and $p<0.05)$. STM treatment obviously increased the expression levels of HO-1 and NQO1 in rat liver as compared with the $\mathrm{CCl}_{4}$ group (Fig. 7).

\section{Discussion}

The main finding of this study was that STM, an iridoid compound from Swertia mussotii Franch (Gentianaceae), was instrumental in attenuating liver injury in rats induced by a chronic $\mathrm{CCl}_{4}$ exposure. In the present study, $\mathrm{CCl}_{4}$ treatment resulted in a significant elevation in serum marker enzymes, ALT, AST and ALP, indicating the liver damage. Besides, severe liver lesion was also induced by $\mathrm{CCl}_{4}$. All of the above-mentioned pathological changes were significantly abrogated with STM treatment, suggesting that STM was able to attenuate $\mathrm{CCl}_{4}{ }_{-}^{-}$ induced liver injury in rats.

$\mathrm{CCl}_{4}$-induced hepatotoxicity was a common animal model for investigating the potential hepatoprotective activity [14]. Lipid peroxidation is one of the principal causes of $\mathrm{CCl}_{4}^{-}$ induced liver injury and is mediated by the free-radical derivatives of $\mathrm{CCl}_{4}$. Lipid peroxidation 
can be monitored by measuring the content of liver MDA. Hepatic antioxidant defense system disruption is characterized by increased lipoperoxides (LPO), MDA and/or altered enzymatic and non-enzymatic antioxidants including SOD, CAT, GPx, GST and GSH. The superoxide radicals are converted to hydrogen peroxide $\left(\mathrm{H}_{2} \mathrm{O}_{2}\right)$ and $\mathrm{O}_{2}$ by the action of SOD, which is further detoxified to water by CAT or GPx. GST plays a physiological role in initiating the detoxification of alkylation of lipid, protein, and nucleic acid, as well as providing cellular resistance to lipid peroxidation by elevating the conjugation of toxic electrophiles with GSH. Thus, the activities of these enzymes have been used to assess oxidative stress in cells [1517]. And the excessive reactive oxygen species (ROS) induced by $\mathrm{CCl}_{4}$ would break the balance between ROS production and antioxidant defense system. In the present study, $\mathrm{CCl}_{4}$ notably decreased the hepatic SOD, CAT, GPx and GST activities, as well as GSH content. Interestingly, STM markedly restored the activities of those antioxidant enzymes and the content of GSH in the liver of $\mathrm{CCl}_{4}$-treated rats. It suggested that STM could attenuate oxidative stress at least partly by renewing the activities of antioxidant enzymes and increasing the content of GSH in liver.

A series factors are believed to be involved in the pathogenesis of the liver injury, including oxidative stress, inflammation and immune reactions. Excessive ROS induced by $\mathrm{CCl}_{4}$ can induce the nuclear factor kappa B (NF- $\left.\kappa \mathrm{B}\right)$ activation and its subsequent nuclear translocation, which are responsible for modulation of liver injury by regulating inflammatory cytokine production, such as TNF- $\alpha$, and the induction of inflammation associated enzymes, including iNOS $[18,19]$. $\mathrm{CCl}_{4}$ exposure generates excessive levels of nitric oxide (NO) through activation of iNOS, thus contributing to damage in hepatic tissue [20]. Hepatic injury in rats treated with $\mathrm{CCl}_{4}$ also developed with an increase in the activity of MPO, an index of hepatic neutrophil infiltration [21]. Our results revealed that STM significantly inhibited MPO and iNOS activity consistent with decreased NO level. In addition, proinflammatory cytokines including IL-1 $\beta$, and IL- 6 and TNF-a have been the focus of investigations of inflammatory organ injury because the uncontrolled and prolonged action of these proteins is potentially harmful [22]. Interestingly, STM significantly reduced inflammatory cytokine levels (IL-1 $\beta$, IL-6 and TNF- $\alpha$ ), thus alleviated inflammatory response in the liver. We preliminarily speculated that STM-mediated hepatoprotection might also benefit from its anti-inflammatory action.

It has been reported that STM treatment could induce detoxification enzymes and efflux transporters in rats [23]. A significant induction of the expression of the basolateral efflux transporters Mrp3 and Mrp4 and canalicular transporter Mdr1 was observed in rats treated with STM compared with the controls. And the expression of detoxification enzymes Cyp3a, Ugt2b, Sult2a1 and Gsta1 in rats treated with STM was also significantly higher than that in the controls. The induced detoxification enzymes thus increase the water solubility of hydrophobic bile acids through hydroxylation (Cyp3a), glucuronidation (Ugt2b), and sulphation (Sult2a1) [24-26]. Generally, $\mathrm{CCl}_{4}$ is metabolized to highly reactive trichloromethyl free radicals mainly by CYP2E1 [27]. On the other hand, the trichloromethyl radicals generated may bind either at the heme group of CYP or at the active site of the enzyme near the heme group, leading to the inactivation of CYP [28]. Total CYP and CYP isoenzyme concentrations were found to be decreased in rats with severe cirrhosis [29]. In acute liver injury induced by $\mathrm{CCl}_{4}$, there were drastic decreases in the activities of the main liver P450 isoenzymes such as CYP1A2, CYP2C6, CYP2E1 and CYP3A2, as well as their protein expressions due to oxidative stress [30]. And chronic $\mathrm{CCl}_{4}$-induced liver injury was also characterized by dysregulation of numerous genes involved in metabolism [31]. In this study, considerable decreases in hepatic CYP3A and CYP2E1 protein level were found in rats treated with $\mathrm{CCl}_{4}$ alone. In contrast, in rats treated with STM, CYP3A and CYP2E1 protein content was less suppressed following $\mathrm{CCl}_{4}$ administration. Our data suggested that STM might restore the hepatic CYP3A and CYP2E1 expression and lead to a rapid recovery from $\mathrm{CCl}_{4}$-induced liver injury. Moreover, bile salt export pump (Bsep) and multidrug resistanceassociated proteins (Mrps) are members of the ATP-binding cassette family of membrane transporters mainly locating in the canalicular membrane of hepatocytes. Bsep mediates the 
concentrative transport of monovalent bile salts into the canaliculus [32]. Mrps transport relatively hydrophilic compounds and drugs [33]. Among them, Mrp2 mainly expresses in hepatocytes acting as a host defense mechanism preventing the influx of harmful substrates from the apical side and facilitating its efflux out of cells. It has been demonstrated that the up-regulation of Bsep and Mrp2 contributed to the reduced hepatic bile acids and the protective effects of alisol B 23-acetate against $\mathrm{CCl}_{4}$-induced hepatotoxicity in mice [34]. The two basolateral transporters Mrp3 and Mrp4 are bile acids efflux transporters that eliminate conjugated bile acids from hepatocytes and readily influenced by liver toxicants also. In $\mathrm{CCl}_{4}$ lesioned rodent model, Mrp3 and Mrp4 were differentially regulated according to animal species and/or experimental methods [35, 36]. Besides, PDZK1 is a PSD-95/discs large/ ZO-1 (PDZ)-based adaptor protein that regulates several membrane-transporting proteins in epithelia [37]. It has been demonstrated that PDZK1 could regulate the expression and function of Mrp2 and Mrp4 [38, 39]. Recently, the expression of PDZK1 was found to be significantly down-regulated during cytokine-induced inflammation [40, 41]. In this study, $\mathrm{CCl}_{4}$ also repressed the protein expression of PDZK1, which was abolished by STM treatment, suggesting a novel regulatory mechanism of hepatic transporters during liver injury. Our results implied that STM could stimulate Bsep and Mrps expression to excrete toxic substances and reduce the hepatotoxicity, highlighting the protective role for STM in liver damage.

The role of the Nrf2/ARE signaling pathway in liver pathophysiology and the potential application of $\mathrm{Nrf} 2$ as a therapeutic target to treat viral hepatitis, alcoholic and nonalcoholic liver disease and fibrosis are well documented [42]. Several natural antioxidants exert hepatoprotective effects not only by scavenging the free radical, but also by augmenting the expression of cytoprotective and/or antioxidant genes via Nrf2 signaling pathway [43]. Normally, Nrf2 is tethered in the cytoplasm by the Kelth-like ECH-associated protein 1 (Keap1) for subsequent proteasomal degradation. Upon stimulation by inducers, Nrf2 dissociates from Keap1 and translocates into the nucleu where it binds to the ARE of target genes coding for a number of antioxidant enzymes and phase II detoxifying enzymes. This will lead to activation of Phase II detoxifying enzymes such as glutathione-S-transferase (GST), quinone oxidoreductase 1 (NQO1), hemeoxygenase-1 (HO-1) that efficiently protect mammalian cells from various toxicants $[44,45]$. In our study, Nrf2 expression was notably increased by STM treatment compared with the $\mathrm{CCl}_{4}$ group, suggesting the activation of Nrf2 defense pathway. And STM treatment obviously enhanced expression of the signaling components, such as HO-1 and NQ01. HO-1 is a rate-limiting enzyme in the catabolism of heme belonging to a family of cytoprotective and detoxification genes that possess AREs in their regulatory regions. Excessive oxidative stress has been suggested as a reason for the upregulation of $\mathrm{HO}-1$, as this enzyme is known to be readily inducible upon such stressors [46]. H0-1 plays a key role in maintaining antioxidant homeostasis and limits hepatotoxicity during chronic liver diseases [47-49]. In our study, the expression of HO-1 protein was significantly increased after $\mathrm{CCl}_{4}$ treatment. And STM treatment further augmented HO-1 protein expression following $\mathrm{CCl}_{4}$ treatment, which suggests that a strong inductive response of HO-1 by STM is to protect hepatocytes from $\mathrm{CCl}_{4}$-induced oxidative cellular injuries. NQO1 is a phase II detoxifying enzyme which can be used for catalyzing the two-electron reduction and detoxification of quinones and other redoxcycling endogenous and exogenous chemicals [50]. In our study, STM treatment also reversed the expression of NQO1 down-regulated by $\mathrm{CCl}_{4}$. These results indicated that the protective effects of STM might link with the activation of the Nrf2 pathway and up-regulation of HO-1 and NQO1.

In conclusion, STM effectively ameliorates the $\mathrm{CCl}_{4}$-induced liver injury and inflammation in rats through alleviation of oxidative stress and induction of hepatic detoxification enzymes and efflux transporters expression, at least in part, via activation of the Nrf2/HO-1 pathway. And there are different signaling pathways, such as the ERK and PI3K/Akt pathways, involved in the beneficial effect of anti-fibrotic natural agent [51]. Further studies on the possible effect of STM on any other cell signaling pathways involved in the pathogenesis of $\mathrm{CCl}_{4}$-induced hepatotoxicity are still ongoing.

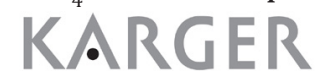




\begin{tabular}{|c|c|}
\hline Cellular Physiology & Cell Physiol Biochem 2017;41:2242-2254 \\
\hline and Biochemistry & 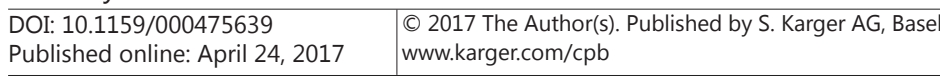 \\
\hline
\end{tabular}

\section{Abbreviations}

ALT (Alanine aminotransferase); AST (Aspartate aminotransferase); ALP (Alkaline phosphatase); $\mathrm{CCl}_{4}$ (Carbon tetrachloride); SOD (Superoxide dismutase); GPx (Glutathione peroxidase); CAT (Catalase); GST (Glutathione S-transferase); GSH (Reduced glutathione); LPO (Lipoperoxides); MDA (Malondialdehyde); ROS (Reactive oxygen species); MPO (myeloperoxidase); iNOS (inducible nitric oxide synthase); NO (nitric oxide); IL-1 $\beta$

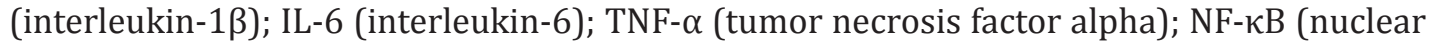
factor kappa B); HO-1 (Hemeoxygenase-1); NQO1 (NAD(P)H: quinone oxidoreductase-1); Nrf2 (Nuclear factor E2-related factor 2); Keap1 (Kelch-like ECH-associated protein-1); ARE (Antioxidant response elements); STM (swertiamarin); H\&E (Hematoxylin and eosin); CYP (Cytochrome P450); Bsep (bile salt export pump); Mrps (multidrug resistance-associated proteins).

\section{Acknowledgements}

This work is financially supported by National Natural Science Foundation of China (81503146).

\section{Disclosure Statement}

None of the authors reports a duality of interest.

\section{References}

1 Jaishree V, Badami S: Antioxidant and hepatoprotective effect of swertiamarin from Enicostemma axillare against D-galactosamine induced acute liver damage in rats. J Ethnopharmacol 2010;130:103-106.

2 Tian C, Zhang T, Wang L, Shan Q Jiang L: The hepatoprotective effect and chemical constituents of total iridoids and xanthones extracted from Swertia mussotii Franch. J Ethnopharmacol 2014;154:259-266.

- Z Zhang L, Cheng Y, Du X, Chen S, Feng X, Gao Y, Li S, Liu L, Yang M, Chen L, Peng Z, Yang Y, Luo W, Wang R, Chen W, Chai J: Swertianlarin, an Herbal Agent Derived from Swertia mussotii Franch, Attenuates Liver Injury, Inflammation, and Cholestasis in Common Bile Duct-Ligated Rats. Evid Based Complement Alternat Med 2015;2015:948376.

-4 Li S, Wang Q Tao Y, Liu C: Swertiamarin Attenuates Experimental Rat Hepatic Fibrosis by Suppressing Angiotensin II-Angiotensin Type 1 Receptor-Extracellular Signal-Regulated Kinase Signaling. J Pharmacol Exp Ther 2016;359:247-255.

5 Jin S, Li H, Han M, Ruan M, Liu Z, Zhang F, Zhang C, Choi Y, Liu B: Mesenchymal Stem Cells with Enhanced Bcl-2 Expression Promote Liver Recovery in a Rat Model of Hepatic Cirrhosis. Cell Physiol Biochem 2016;40:1117-1128.

-6 Basu S: Carbon tetrachloride-induced lipid peroxidation: eicosanoid formation and their regulation by antioxidant nutrients. Toxicology 2003;189:113-127.

7 Manibusan MK, Odin M, Eastmond DA: Postulated carbon tetrachloride mode of action: a review. J Environ Sci Health C Environ Carcinog Ecotoxicol Rev 2007;25:185-209.

-8 Planaguma A, Claria J, Miquel R, Lopez-Parra M, Titos E, Masferrer JL, Arroyo V, Rodes J: The selective cyclooxygenase-2 inhibitor SC-236 reduces liver fibrosis by mechanisms involving non-parenchymal cell apoptosis and PPARgamma activation. FASEB J 2005;19:1120-1122.

-9 Kensler TW, Wakabayashi N, Biswal S: Cell survival responses to environmental stresses via the Keap1Nrf2-ARE pathway. Annu Rev Pharmacol Toxicol 2007;47:89-116.

10 Boettler U, Volz N, Teller N, Haupt LM, Bakuradze T, Eisenbrand G, Bytof G, Lantz I, Griffiths LR, Marko D: Induction of antioxidative Nrf2 gene transcription by coffee in humans: depending on genotype? Mol Biol Rep 2012;39:7155-7162. 


\section{Cellular Physiology Cell Physiol Biochem 2017;41:2242-2254 \begin{tabular}{l|l} 
DOI: 10.1159/000475639 & $\begin{array}{l}\text { O 2017 The Author(s). Published by S. Karger AG, Basel } \\
\text { www.karger.com/cpb }\end{array}$ \\
\hline
\end{tabular}}

Wu et al.: Swertiamarin Protects Against Hepatotoxicity

11 Ma B, Wang J, Tong J, Zhou G, Chen Y, He J, Wang Y: Protective effects of Chaenomeles thibetica extract against carbon tetrachloride-induced damage via the MAPK/Nrf2 pathway. Food Funct 2016;7:1492-1500.

12 Zhuang X, Deng ZB, Mu J, Zhang L, Yan J, Miller D, Feng W, McClain CJ, Zhang HG: Ginger-derived nanoparticles protect against alcohol-induced liver damage. J Extracell Vesicles 2015;4:28713.

13 Cho I, Kim J, Jung J, Sung S, Kim J, Lee N, Ku S: Hepatoprotective effects of hoveniae semen cum fructus extracts in ethanol intoxicated mice. J Exerc Nutrition Biochem 2016;20:49-64.

14 Delire B, Starkel P, Leclercq I: Animal Models for Fibrotic Liver Diseases: What We Have, What We Need, and What Is under Development. J Clin Transl Hepatol 2015;3:53-66.

-15 Martin-Aragon S, de las Heras B, Sanchez-Reus MI, Benedi J: Pharmacological modification of endogenous antioxidant enzymes by ursolic acid on tetrachloride-induced liver damage in rats and primary cultures of rat hepatocytes. Exp Toxicol Pathol 2001;53:199-206.

16 Xu Z, Wang Z, Li JJ, Chen C, Zhang PC, Dong L, Chen JH, Chen Q, Zhang XT, Wang ZL: Protective effects of selenium on oxidative damage and oxidative stress related gene expression in rat liver under chronic poisoning of arsenic. Food Chem Toxicol 2013;58:1-7.

17 Wunjuntuk K, Kettawan A, Charoenkiatkul S, Rungruang T: Parboiled Germinated Brown Rice Protects Against CCl4-Induced Oxidative Stress and Liver Injury in Rats. J Med Food 2016;19:15-23.

18 Ma JQ, Ding J, Zhang L, Liu CM: Ursolic acid protects mouse liver against CCl4-induced oxidative stress and inflammation by the MAPK/NF-kappaB pathway. Environ Toxicol Pharmacol 2014;37:975-983.

19 Ma JQ, Li Z, Xie WR, Liu CM, Liu SS: Quercetin protects mouse liver against CCl(4)-induced inflammation by the TLR2/4 and MAPK/NF-kappaB pathway. Int Immunopharmacol 2015;28:531-539.

20 Tipoe GL, Leung TM, Liong E, So H, Leung KM, Lau TY, Tom WM, Fung ML, Fan ST, Nanji AA: Inhibitors of inducible nitric oxide (NO) synthase are more effective than an NO donor in reducing carbon-tetrachloride induced acute liver injury. Histol Histopathol 2006;21:1157-1165.

21 Ohta Y, Imai Y, Matsura T, Kitagawa A, Yamada K: Preventive effect of neutropenia on carbon tetrachlorideinduced hepatotoxicity in rats. J Appl Toxicol 2006;26:178-186.

22 Shin DS, Kim KW, Chung HY, Yoon S, Moon JO: Effect of sinapic acid against carbon tetrachloride-induced acute hepatic injury in rats. Arch Pharm Res 2013;36:626-633.

-23 Feng XC, Du X, Chen S, Yue D, Cheng Y, Zhang L, Gao Y, Li S, Chen L, Peng Z, Yang Y, Luo W, Wang R, Chen W, Chai J: Swertianlarin, isolated from Swertia mussotii Franch, increases detoxification enzymes and efflux transporters expression in rats. Int J Clin Exp Pathol 2015;8:184-195.

24 Trauner M, Meier PJ, Boyer JL: Molecular pathogenesis of cholestasis. N Engl J Med 1998;339:1217-1227.

25 Zollner G, Marschall HU, Wagner M, Trauner M: Role of nuclear receptors in the adaptive response to bile acids and cholestasis: pathogenetic and therapeutic considerations. Mol Pharm 2006;3:231-251.

26 Wagner M, Zollner G, Trauner M: New molecular insights into the mechanisms of cholestasis. J Hepatol 2009;51:565-580.

27 Recknagel RO, Glende EA, Jr., Dolak JA, Waller RL: Mechanisms of carbon tetrachloride toxicity. Pharmacol Ther 1989;43:139-154.

28 Fernandez G, Villarruel MC, de Toranzo EG, Castro JA: Covalent binding of carbon tetrachloride metabolites to the heme moiety of cytochrome P-450 and its degradation products. Res Commun Chem Pathol Pharmacol 1982;35:283-290.

-29 Bastien MC, Leblond F, Pichette V, Villeneuve JP: Differential alteration of cytochrome P450 isoenzymes in two experimental models of cirrhosis. Can J Physiol Pharmacol 2000;78:912-919.

- 30 Xie Y, Hao H, Wang H, Guo C, Kang A, Wang G: Reversing effects of lignans on CCl4-induced hepatic CYP450 down regulation by attenuating oxidative stress. J Ethnopharmacol 2014;155:213-221.

-31 Mukhopadhyay P, Rajesh M, Cao Z, Horvath B, Park O, Wang H, Erdelyi K, Holovac E, Wang Y, Liaudet L, Hamdaoui N, Lafdil F, Hasko G, Szabo C, Boulares AH, Gao B, Pacher P: Poly (ADP-ribose) polymerase-1 is a key mediator of liver inflammation and fibrosis. Hepatology 2014;59:1998-2009.

-32 Gerloff T, Geier A, Stieger B, Hagenbuch B, Meier PJ, Matern S, Gartung C: Differential expression of basolateral and canalicular organic anion transporters during regeneration of rat liver. Gastroenterology 1999;117:1408-1415.

-33 Keppler D: Multidrug resistance proteins (MRPs, ABCCs): importance for pathophysiology and drug therapy. Handb Exp Pharmacol 2011;10.1007/978-3-642-14541-4_8299-323. 


\section{Cellular Physiology Cell Physiol Biochem 2017;41:2242-2254 \begin{tabular}{ll|l} 
and Biochemistry Published onlIne: AprII 24, 2017 & $\begin{array}{l}\text { C } 2017 \text { The Author(s). Published by S. Karger AG, Basel } \\
\text { www.karger.com/cpb }\end{array}$ \\
\hline
\end{tabular}}

Wu et al.: Swertiamarin Protects Against Hepatotoxicity

-34 Meng Q, Chen X, Wang C, Liu Q, Sun H, Sun P, Huo X, Liu Z, Liu K: Protective effects of alisol B 23-acetate from edible botanical Rhizoma alismatis against carbon tetrachloride-induced hepatotoxicity in mice. Food Funct 2015;6:1241-1250.

-35 Aleksunes LM, Scheffer GL, Jakowski AB, Pruimboom-Brees IM, Manautou JE: Coordinated expression of multidrug resistance-associated proteins (Mrps) in mouse liver during toxicant-induced injury. Toxicol Sci 2006;89:370-379.

-36 Okumura H, Katoh M, Minami K, Nakajima M, Yokoi T: Change of drug excretory pathway by CCl4-induced liver dysfunction in rat. Biochem Pharmacol 2007;74:488-495.

- 37 Kocher O, Comella N, Gilchrist A, Pal R, Tognazzi K, Brown LF, Knoll JH: PDZK1, a novel PDZ domaincontaining protein up-regulated in carcinomas and mapped to chromosome 1q21, interacts with cMOAT (MRP2), the multidrug resistance-associated protein. Lab Invest 1999;79:1161-1170.

-38 Emi Y, Nomura S, Yokota H, Sakaguchi M: ATP-binding cassette transporter isoform C2 localizes to the apical plasma membrane via interactions with scaffolding protein. J Biochem 2011;149:177-189.

-39 Park J, Kwak JO, Riederer B, Seidler U, Cole SP, Lee HJ, Lee MG: Na(+)/H(+) exchanger regulatory factor 3 is critical for multidrug resistance protein 4-mediated drug efflux in the kidney. J Am Soc Nephrol 2014;25:726-736.

40 Lenzen H, Lunnemann M, Bleich A, Manns MP, Seidler U, Jorns A: Downregulation of the NHE3-binding PDZ-adaptor protein PDZK1 expression during cytokine-induced inflammation in interleukin-10-deficient mice. PLoS One 2012;7:e40657.

41 Yeruva S, Chodisetti G, Luo M, Chen M, Cinar A, Ludolph L, Lunnemann M, Goldstein J, Singh AK, Riederer B, Bachmann O, Bleich A, Gereke M, Bruder D, Hagen S, He P, Yun C, Seidler U: Evidence for a causal link between adaptor protein PDZK1 downregulation and $\mathrm{Na}(+) / \mathrm{H}(+)$ exchanger NHE3 dysfunction in human and murine colitis. Pflugers Arch 2015;467:1795-1807.

-42 Shin SM, Yang JH, Ki SH: Role of the Nrf2-ARE pathway in liver diseases. Oxid Med Cell Longev 2013;2013:763257.

-43 Al-Rasheed NM, Fadda LM, Ali HM, Abdel Baky NA, El-Orabi NF, Al-Rasheed NM, Yacoub HI: New mechanism in the modulation of carbon tetrachloride hepatotoxicity in rats using different natural antioxidants. Toxicol Mech Methods 2016;26:243-250.

-44 Huang HC, Nguyen T, Pickett CB: Phosphorylation of Nrf2 at Ser-40 by protein kinase C regulates antioxidant response element-mediated transcription. J Biol Chem 2002;277:42769-42774.

45 Farombi EO, Shrotriya S, Na HK, Kim SH, Surh YJ: Curcumin attenuates dimethylnitrosamine-induced liver injury in rats through Nrf2-mediated induction of heme oxygenase-1. Food Chem Toxicol 2008;46:12791287.

-46 Petrache I, Otterbein LE, Alam J, Wiegand GW, Choi AM: Heme oxygenase-1 inhibits TNF-alpha-induced apoptosis in cultured fibroblasts. Am J Physiol Lung Cell Mol Physiol 2000;278:L312-319.

-47 Nakahira K, Takahashi T, Shimizu H, Maeshima K, Uehara K, Fujii H, Nakatsuka H, Yokoyama M, Akagi R, Morita K: Protective role of heme oxygenase-1 induction in carbon tetrachloride-induced hepatotoxicity. Biochem Pharmacol 2003;66:1091-1105.

-48 Yao P, Nussler A, Liu L, Hao L, Song F, Schirmeier A, Nussler N: Quercetin protects human hepatocytes from ethanol-derived oxidative stress by inducing heme oxygenase-1 via the MAPK/Nrf2 pathways. J Hepatol 2007;47:253-261.

49 Domitrovic R, Jakovac H, Marchesi VV, Sain I, Romic Z, Rahelic D: Preventive and therapeutic effects of oleuropein against carbon tetrachloride-induced liver damage in mice. Pharmacol Res 2012;65:451-464.

-50 Su C, Xia X, Shi Q, Song X, Fu J, Xiao C, Chen H, Lu B, Sun Z, Wu S, Yang S, Li X, Ye X, Song E, Song Y: Neohesperidin Dihydrochalcone versus CCl(4)-Induced Hepatic Injury through Different Mechanisms: The Implication of Free Radical Scavenging and Nrf2 Activation. J Agric Food Chem 2015;63:5468-5475.

-51 Lin X, Bai F, Nie J, Lu S, Lu C, Zhu X, Wei J, Lu Z, Huang Q: Didymin Alleviates Hepatic Fibrosis Through Inhibiting ERK and PI3K/Akt Pathways via Regulation of Raf Kinase Inhibitor Protein. Cell Physiol Biochem 2016;40:1422-1432. 